\title{
A REVIEW OF ESSENTIAL TECHNOLOGIES FOR AUTONOMOUS AND SEMI- AUTONOMOUS ARTICULATED HEAVY vehicles
}

\author{
Amir Rahimi*, Yuping He \\ Department of Automotive, Mechanical and Manufacturing, Ontario Tech University, Oshawa, Canada \\ *amir.rahimi@ontariotechu.ca
}

\begin{abstract}
To increase the safety of articulated heavy vehicles (AHVs), attention has been paid to exploring active vehicle safety systems (AVSSs), e.g., anti-lock braking systems. These active vehicle safety technologies are classified as 'reactive safety systems', designed to react to the current vehicle state. These systems are effective, but do not consider the effect of driver error. The main cause of traffic accidents is linked to human errors. A resolution to the problem is autonomous driving, which removes human factors from the control loop. There will be a transition period, during which most vehicles have some capabilities of autonomous driving. Since the late 1990s, lane departure warning and adaptive cruise control systems have been proposed. These technologies are classified as 'predictive safety systems' (PSSs), considering not only the current vehicle state, but also the predicted vehicle state and environmental hazards. For passenger vehicles, several PSSs have been investigated. These PSSs are featured with semiautonomous driving functions. AHVs represent a 7.5 times higher risk than passenger cars in highway operations. However, much less attention has been paid to exploring the PSSs for AHVs. This paper reviews the current status of essential technologies proposed and examined for autonomous and semi-autonomous AHVs. The pros and cons of the technologies are discussed and analyzed. As a result of the review, future research efforts are identified.
\end{abstract}

Keywords-autonomous; semi-autonomous; state-of-the-art automated driving technologies; articulated heavy vehicle.

\section{INTRODUCTION}

The automotive industry is moving towards a new era of autonomous ground vehicles (AGVs). The term AGV refers to a car that can do the navigation during driving through a highway or city traffic and even off-road paths without human intervention. With software, computers, sensors and connected networks being increasingly used, the design of such vehicles is undergoing significant changes.

Worldwide around 1.25 million people are killed per year in road vehicle accidents [1]. Table 1 demonstrates statistical data regarding the car crashes only in the US and Canada in 2016. 93 per cent of serious crashes are because of human errors. Considering the fact that in 2016 more than 37,000 people were killed in around 32,000 fatal motor vehicle accidents in North America [2], and also economic costs of crashes are unbelievably high (277 billion dollars in the US), the lifesaving and financial benefits of driving assistance technologies become undeniable. AGVs have the potential to decline human errors from the accidents, thereby leading considerably protecting passengers, drivers and pedestrians and reducing the financial costs. Utilizing Advanced Driver Assistance Systems (ADAS), e.g. Adaptive Cruise Control (ACC), Traffic Jam Assistant (TJA) and Collision Avoidance Systems, fatal car crashes decreased in Europe between 2001 and 2015 by $48 \%$ [3]. Figure 1 presents history of driving assistance functions application and their potential future evolution.

TABLE I. THE U.S. AND CANADA MOTOR VEHICLE CRASHES AND HUMAN FACTOR INVOLVEMENT [2][4]

\begin{tabular}{|l|c|c|}
\hline \multicolumn{1}{|c|}{ criteria } & U.S. & Canada \\
\hline Total crashes per year & 5.5 million & 290,000 \\
\hline Human causes as main factor & $93 \%$ & $80 \%$ \\
\hline Economic costs of crashes & $\$ 277$ billion & $\$ 37$ billion \\
\hline Total fatal and injurious crashes per year & 2.22 million & 165,140 \\
\hline Fatal crashes per year & 32,367 & 1,895 \\
\hline $\begin{array}{l}\text { Total fatal and injurious crashes per year } \\
\text { involving a heavy truck }\end{array}$ & 96129 & 12000 \\
\hline
\end{tabular}

As the safety merits of automated driving are paramount, governments urgently need to develop vehicle safety guidelines for the design of AGVs [5]. The guidelines are expected to identify design aspects for manufacturers to consider when developing, testing and deploying such vehicles. Recently, many design methods for autonomous vehicles have been proposed, but the literature focuses on semiautonomous and autonomous driving of single-unit vehicles, e.g., cars. Heavy vehicles exhibit unique lateral dynamic characteristics. For example, the static roll-over limit for heavy trucks can be as low as $0.35 \mathrm{~g}$, whereas the rollover limit for passenger cars is typically 1.1g [6]. In 1997, the US National Highway Traffic Safety Administration reported more than 15,000 rollover accidents of commercial vehicles, of which 9400 were rollovers of AHVs [7]. On the other hand, mortality due to AHV accidents in the US in 2017 rose to 5.8 per cent compared with 2016 [8]. However, little attention has been paid to autonomous driving for these large vehicles. 


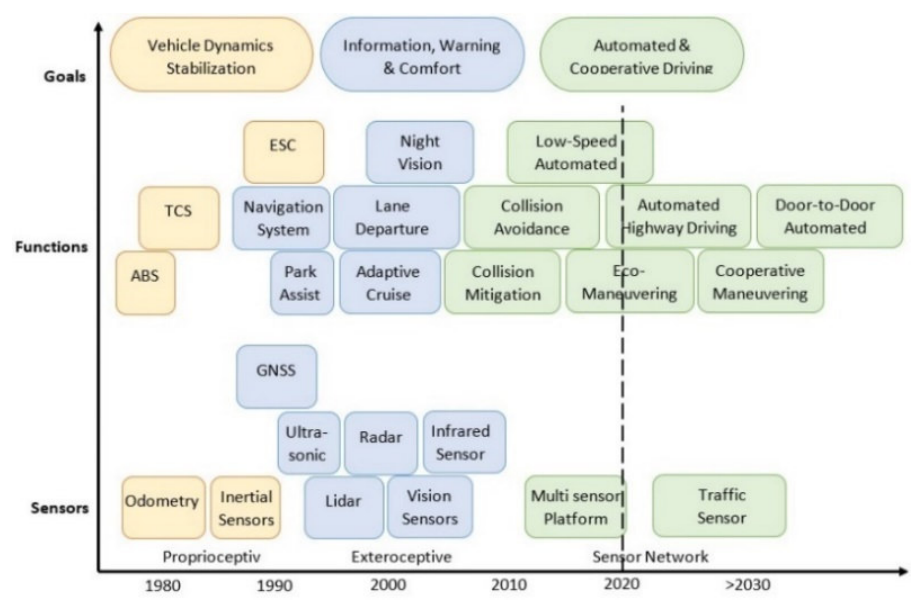

Figure 1. Past and future evolution of driving assistance functions from market perspective, reproduced from [9]

To increase vehicle safety, AVSSs, e.g., vehicle stability control, have been commercialized [10]. These AVSSs can be classified as reactive safety systems (RSSs), designed to react to the current vehicle state [11]. Although RSSs are effective in increasing safety, they do not consider the effect of driver mistakes. As noted earlier, human errors cause the vast majority of traffic collisions [5], and the potential resolution to the human error problem is autonomous driving [12], removing human factors from the control loop. The mass deployment of autonomous driving systems has been hindered due to the lack of formal approaches for verifying the safety of such systems in arbitrary situations [7][8]. Automated driving industry has come a long distance so far, and still there will be a long transition period, in which most vehicles have some capabilities of autonomous driving. Figure 2 depicts a brief timeline of vehicles automated driving and safety functions development. Since the late 1990s, advanced driver assistance systems, e.g., lane departure prevention, have been developed. These systems are classified as 'predictive safety systems' (PSSs), considering not only current vehicle state, but also predicted vehicle state and hazards. The last two decades have witnessed extensive research of semi-autonomous vehicles, which are human driven vehicles with autonomous driving capabilities [13]. These vehicles are level 2/3 automated vehicles [14].

To date the research activities in semi-autonomous and autonomous driving have mainly been dedicated to passenger cars. Heavy goods vehicles represent a 7.5 times higher risk than passenger cars in highway operations [15]. However, much less attention has been paid to exploring these PSSs for articulated vehicles and in particular, AHVs. Recently, few studies tackled autonomous driving for articulated construction vehicles [16]-[18], articulated vehicles with automated reverse parking [17], and construction truck [18]. These autonomous systems were designed only considering low-speed trajectory planning and tracking based on kinematic control, neglecting the high-speed dynamic behaviors of articulated vehicles, e.g. trailer sway, jackknifing, and rollover. The interactions of human-machine have been explored for improving the passive steering of articulated vehicles [19][20]. But the interactions have not been considered in autonomous steering. In the literature, there is no published study on semi-autonomous driving for articulated vehicles and, especially, AHVs [6].

This paper reviews some of the academic researches and technological developments in the area of autonomous and semi-autonomous heavy vehicles with a focus on AHVs over the recent years. Section 2 briefly introduces the required background knowledge for AHVs and autonomy in general. Section 3 will describe the path following as the main strategy in automated driving and the relevant control strategies employed. Section 4 reviews the sensor technology needed for autonomous driving. In Section 5 some future directions regarding autonomous and semi-autonomous AHVs will be described. Finally, conclusions of current study are drawn in Section 6.

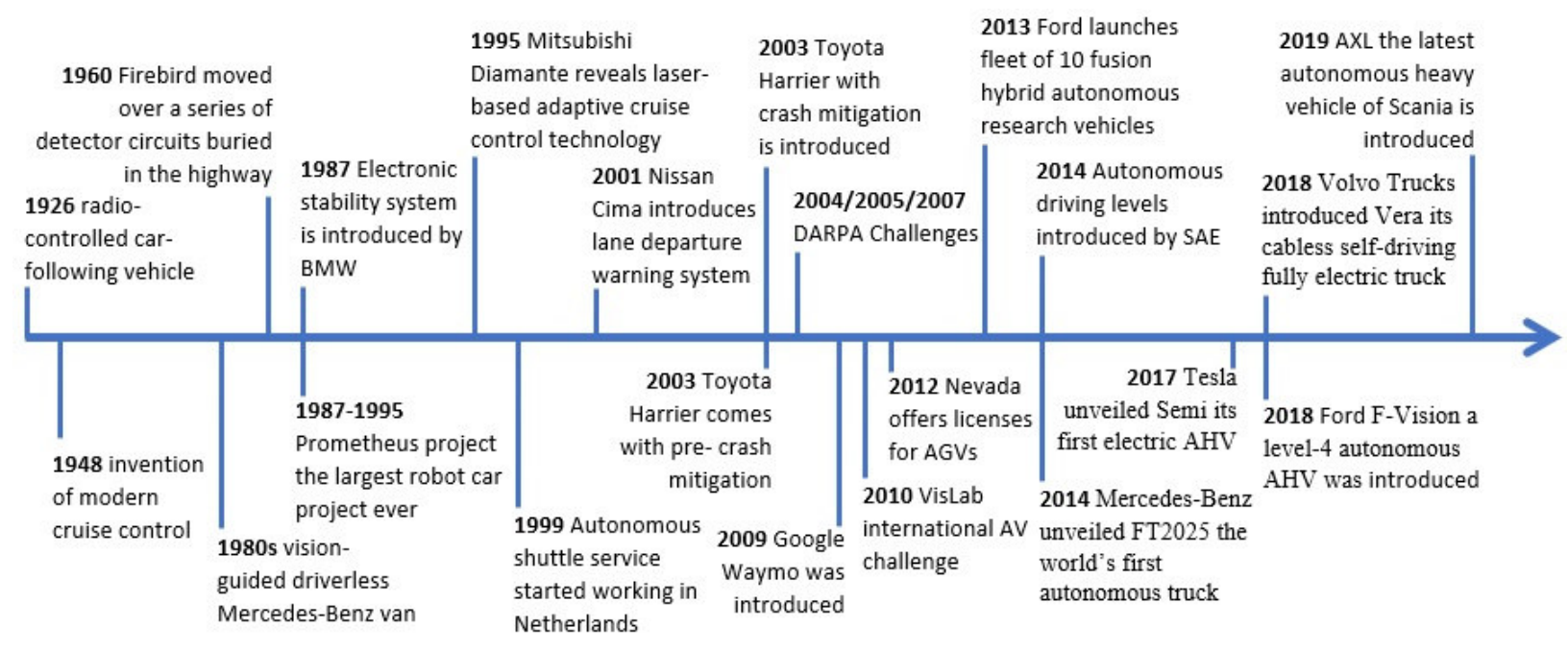

Figure 2. Vehicles automated driving timeline 


\section{BACKGROUND}

\section{A. Autonomy classification and necessary modules}

The ground vehicle automation needs a standard set of terminology and regulations with a taxonomy and definitions to coordinate all the efforts made in this field. In 2014 SAE International introduced new standard J3016 to facilitate collaboration and simplify communication within technical and policy domains. Figure 3 summarizes the levels of driving automation based on this standard.

\begin{tabular}{|c|c|c|c|c|c|}
\hline \multirow[b]{2}{*}{ Level } & \multirow[b]{2}{*}{ Name } & \multicolumn{2}{|c|}{ Dynamic Driving Task (DDT) } & \multirow[b]{2}{*}{$\begin{array}{c}D D T \\
\text { Fallback }\end{array}$} & \multirow{2}{*}{$\begin{array}{c}\text { Operational } \\
\text { Design Domain } \\
\text { (ODD) }\end{array}$} \\
\hline & & $\begin{array}{c}\text { Sustained lateral and } \\
\text { Iongitudinal vehicle } \\
\text { motion control }\end{array}$ & $\begin{array}{l}\text { Object and Event } \\
\text { Detection and } \\
\text { Response (OEDR) }\end{array}$ & & \\
\hline \multicolumn{6}{|c|}{ Driver performs part or all of the DDT } \\
\hline 0 & No Driving Automation & Driver & Driver & Driver & $N / A$ \\
\hline 1 & Driver Assistance & Driver and System & Driver & Driver & Limited \\
\hline 2 & Partial Driving Automation & System & Driver & Driver & Limited \\
\hline \multicolumn{6}{|c|}{ Automated Driving System (ADS "System") performs the entire DDT (while engaged) } \\
\hline 3 & $\begin{array}{l}\text { Condiltional Driving } \\
\text { Automation }\end{array}$ & System & System & $\begin{array}{c}\text { Falliback-ready } \\
\text { user }\end{array}$ & Limited \\
\hline 4 & High Driving Automation & System & System & System & Limited \\
\hline 5 & Full Driving Automation & System & System & System & Unlimited \\
\hline
\end{tabular}

Figure 3. Levels of driving automation, reproduced from [14]

According to [21], an autonomous vehicle system accounts for three basic modules.

- Sensing and prediction: to prepare real time data to make the system capable to recognize instantaneous vehicle location and surrounding environment and produce row data for the system to process.

- Planning: to utilize the provided data to design the safe and feasible path to follow

- Control: to adopt appropriate control strategies to lead the vehicle to the desired path.

After designing the appropriate control strategy, actuators work as the final stage of automated driving. In a conventional vehicle driver adjusts the speed by depressing the gas pedal or braking pedal, and turning the steering wheel to drive the vehicle in the correct and safe path. Differently, autonomous vehicles need some actuators to convert the input electrical signals from the controller to mechanical movements to control the steering, engine throttle and so on.

\section{B. Types of $A H V S$}

As mentioned earlier an AHV is a combination of two or more rigid vehicles units, which are connected to each other by mechanical couplings, termed hitches, at articulation points. Figure 4 shows important units of AHV [22].

The two most popular AHVs use just one trailer, i.e., the tractor-semitrailer and the truck-full trailer. Multi-trailer combinations are usually formed by connecting one or more additional trailers to a tractor-semitrailer combination. Combinations with more than one trailer are often named vehicle trains. They are referred to as A-, B-, or C-trains relying on the type of coupling between trailers [22]. Figure 5 illustrates the three configurations.

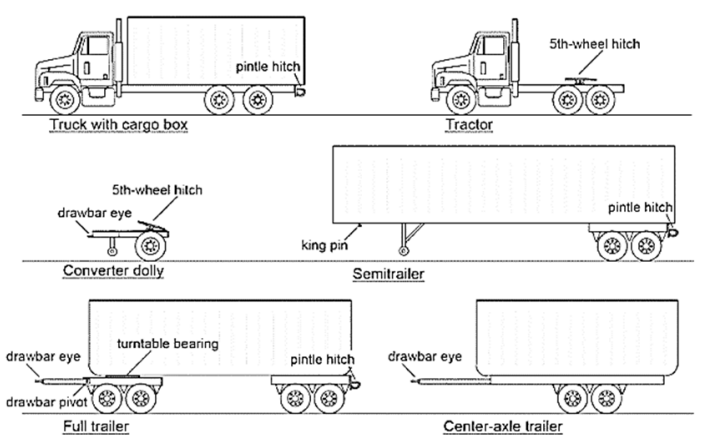

Figure 4. Different types of vehicle units and couplings used in AHVs [22]

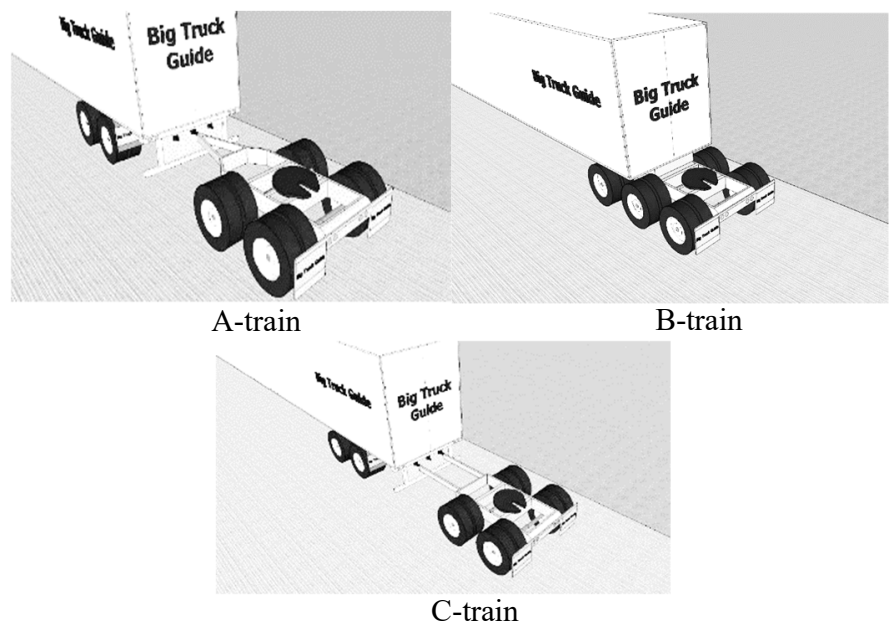

Figure 5. different ways that two trailers are hooked to one another in North America [23].

\section{Maneuvering characteristics of $A H V S$}

Some regulations for AHVs are established based on performance-based standards (PBSs). These regulations incorporate specific performance criteria in which required level of performance is quantified [6]. One instance of performance-based characteristics defined for AHVs movement in lateral and longitudinal directions can be found in [24] and are classified in two categories.

- The characteristics for longitudinal direction including startability, gradeability, acceleration capability, stopping distance, and down-grade holding capability.

- The characteristics for lateral direction comprising rearward amplification (RWA), swept path width (SPW), high-speed transient off-tracking (HSTO), high-speed steady-state off- tracking (HSSO), yaw damping coefficient (YDC), straight line off-tracking (SLO), lateral clearance time (LCT), steady-state rollover threshold (SRT), and deceleration capability in a turn.

The most significant lateral characteristics for high-speed maneuvering are RWA, HSTO, HSSO and YDC, which are described as follows [6]:

- RWA is the relationship between the maximum motion of the first and last vehicle units during a specified steering maneuver and vehicle speed. It 
denotes the increased risk of a semi-trailer roll-over or swing-out. The maximum rearward amplification allowed by the Australian PBS is 5.7 times the static rollover threshold expressed in g's where $\mathrm{g}=9.81$ $\mathrm{m} / \mathrm{s}^{2}$.

- The off-tracking characteristics, HSTO and HSSO, describe the lateral deviation between the path of the front axle and the path of the most severely offtracking axle of the semi-trailer. Examples of HSTO and HSSO are given in Figures 6 and 7. These measures indicate the additional space needed for the semi-trailer in a specific steering maneuver and vehicle speed.

- $\quad$ The YDC is the damping ratio of the least damped articulation joint's angle during free-yaw oscillations of the vehicle combination, after a specific steering maneuver and vehicle speed. A longer decay time might result in higher driver workload and increased risk of the safety of other road users.

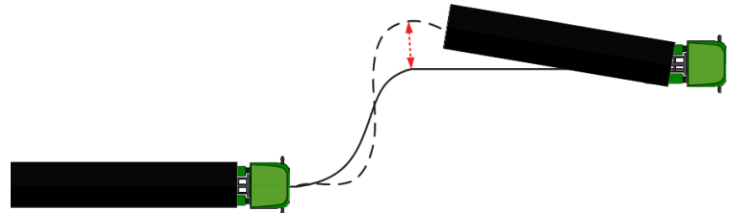

Figure 6. Lane-change maneuver illustrating HSTO, RA and YDC [6]

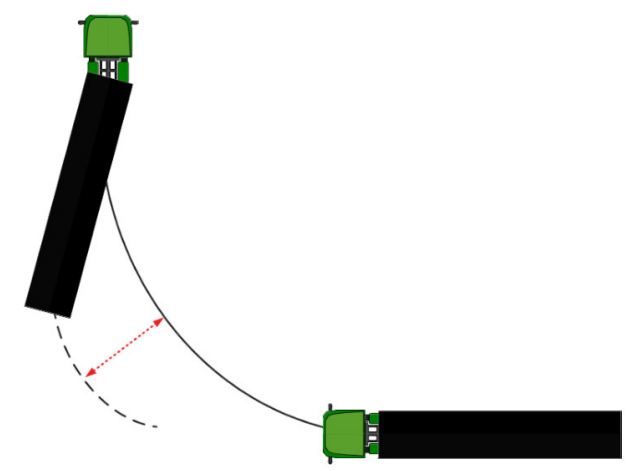

Figure 7. Steady-state maneuver illustrating HSSO [6]

D. Vehicle models used in automated driving controller design

During the decades of vehicle lateral dynamics studies, linear handling model known as bicycle model has been used predominantly. Vehicle models usually presume the vehicle body as a rigid body with concentrated sprung mass at the center of gravity. A commonly used model that only considers lateral and yaw motions is known as a handling model. There are three major vehicle models including geometric, kinematic and dynamic models. In geometric model, only the geometrical dimensions of the vehicle are considered. Kinematic model just considers the motion of vehicle in terms of acceleration, velocity and position. Dynamic model, differently, considers the vehicle motion in terms of its internal forces, inertia and energy properties.

Models used for tractor-semitrailers vary in a vast range of complexity from large multi-body combinations with numerous degrees of freedom, which demand costly calculations compared against simple 3-DOF bicycle models [25]. In many of the published research works such as [7], [26]-[28] a simplified 5-DOF, 3-wheel linear model has been used. This simple model seems quite sufficient for analyzing lateral stability of a tractor-semitrailer in path following control strategy design which is an integral part of any autonomous AHV design. Figure 8 shows a scheme of this model.

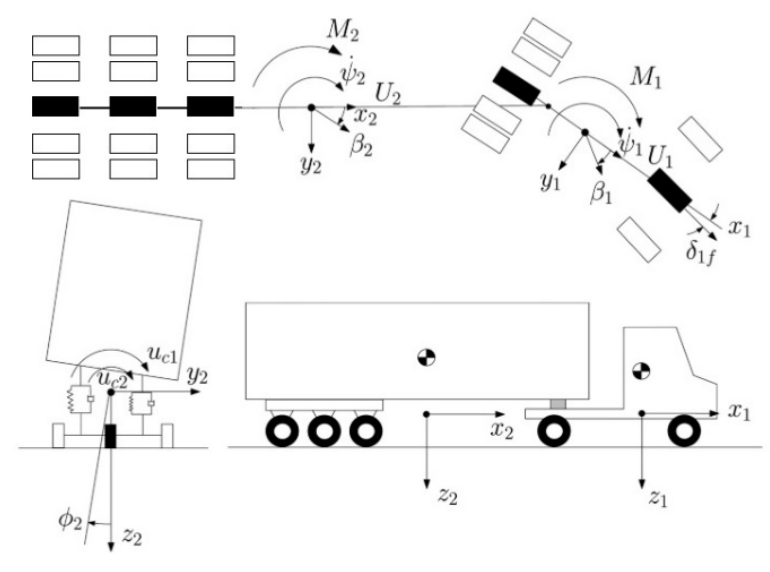

Figure 8. Schematic representation of the AHV model

\section{E. Advantages of autonomous AHVS and barriers to implementation}

Table 1 highlights the magnitude of motor vehicle crashes in North America and indicates that a majority number of crashes happen because of human errors including inattention, distraction, or speeding. Besides, it denotes that a considerable part of the fatal crashes involves heavy vehicles comprising AHVs. As a result, the more the heavy vehicle utilize autonomy, the less fatal crashes would occur. Poor weather, such as fog and snow, is another challenging situation for drivers, which can be tackled by using sensors and artificial intelligence as an essential part of automated driving in AHVs. Apart from making AHVs safer, researchers believe using just ACC function as a primitive feature of autonomy can increase fuel economy and traffic congestion by $23-39 \%$ and $8-13 \%$ respectively, for all vehicles in the highway travel stream [29]. Furthermore, it is estimated that $5-20 \%$ of all driving is either autonomous or semi-autonomous by 2025 and the resulting global financial impact would be $\$ 200$ billion to $\$ 1.9$ trillion [30].

The first barrier on the way of large-scale market of autonomous AHVs is the cost, due to the technology that requires additional new sensors, communication networks, navigation devices, and specific software design. For example, LiDAR systems on top of other sensors cost up to $\$ 70,000$ for long-range LiDAR, which seems to be vital at least for AHVs current automated driving technology. Other issues include lack of legislations pertaining driving autonomous AHVs, restrictions in giving certification to AVs, arising insurance and liability concerns and worries about electronic security, computer hackers and terrorist applications [29]. 


\section{PATH FOLLOWING AND LATERAL CONTROL OF AHVS}

There are two main goals for AHVs control within an autonomous and semi-autonomous driving [31]. To control both the longitudinal and lateral dynamics using the embedded control system with sophisticated software that are actually codes written to do the job. The control algorithms involve a higher level of control (strategy control) and a lower level of control (vehicle control). The former makes decisions based on the data received from the infrastructure and other vehicles by which are affected through maneuver. The later includes vehicle steering, throttle and brake control.

Longitudinal dynamics control of AHVs mainly refers to regulating vehicle speed in order to retain enough space between vehicles. For implementing a successful longitudinal control four types of data are needed. Speed and acceleration of the host vehicle, speed and acceleration of the preceding vehicle, the distance from the leading vehicle and in the case of platooning speed and acceleration of the first vehicle.

Lateral control of AHVs is to drive the vehicle close to the center of the desired lane, which includes not only straight road section, but also curved path, and roundabout way (lanekeeping maneuver). Under such complicated operating conditions, rollover is a common accident that AHVs may experience during lane changes or cornering maneuvers, which often causes harsh results, e.g., considerable financial costs and fatalities. This is usually because of higher center of gravity (CG) of heavy vehicles compared with passenger cars. Hence, effective control strategies like active suspension control, active steering and active braking should be employed to increase the roll stability.

Path following is a vital issue for autonomous driving. The path following control for AGVs involves maneuvering the vehicle autonomously through the steering control. This targets to help the vehicle follow the desirable path defined by the navigation system via sensors. There are different strategies for the lateral control of AHVs during path following. Some researchers proposed the use of an active trailer steering system to enhance the path following and stability control of AGVs.

For example, in a study [32] a model predictive controller was utilized to make the vehicle follow a path and yaw angle predicted for the trailer while minimizing the side slip angle for different vehicle conditions. The designed controller efficiently improved the lateral stability and off-tracking of the trailer through numerical simulations. In another study [33] a LQR-based active trailer steering controller designed to improve tractor-semitrailer lateral stability at high speeds and the maneuverability in low speeds. The researchers used a 3DOF linear model and a simulated annealing particle swarm optimization algorithm based on TruckSim-Simulink environment and the results were interesting.

A lateral-longitudinal control method was proposed to avoid jackknifing during automated steering [34]. The jackknifing was prevented by employing an anti-windup mechanism in that controlled the articulation angle from exceeding the limit. Reference [35] reported a research work, in which a control strategy adopted to make both tractor and semitrailer of an AHV follow different paths at different vehicle speeds and in the presence of external disturbances. They used nonlinear kinematics-based controller for low speed, while it was not appropriate for high speeds because of high changes in side-slip characteristics. Hence, they combined both low and high-speed controllers using a speeddependent gain in mid speed range. The simulation results proved an improvement in maneuverability at low speeds and an enhancement of stability at high speeds.

In another research work [36], a new lane-keeping controller was introduced to keep an AHV position (lateral and angular) aligned with the lane, as well as maintaining its stability in critical situation. They utilized an optimal control technique and a fuzzy supervisory strategy to adapt the controller to the various driving behaviors of drivers. The system was basically a human driven AHV which reacted appropriately in a case that the AHV deviated from the desired path. Different testing maneuvers were used to show the effectiveness of the controllers designed.

A controller design was proposed to deal with the path following issue for articulated robotic vehicles, which had been equipped with a number of off-axle hitched trailers [37]. The controller was highly scalable nonlinear cascade-like that did not require to set the shortest distance to an ideal path. Instead, it used a segment-platooning reference path (introduced by the researchers) to ensure asymptotic path following. Empirical results exhibited the small sensitivity of designed controller to the parameters uncertainties.

In another research study [38], a new sliding mode control (SMC) strategy was introduced to do the trajectory tracking for articulated vehicles. The designed controller targeted to attain better tracking capability while minimizing the tracking error and declining the chattering phenomenon. The SMC was derived based on a nonlinear kinematic model of the articulated vehicle, and the stability of the control strategy was tested using the Lyapunov's stability method. Finally, the investigators evaluated their controller performance in various paths scenarios using a small-scale model.

As for the rollover prevention in heavy vehicles, reference [39] documented a combined active anti-roll bar (AARB) and active braking controller. Reference [40] introduced a linear quadratic static output feedback control strategy using both AARB and electronic stability program to handle the rollover issue more efficiently.

In one research study [41], a MPC-based control strategy was designed to improve the roll stability in a path following maneuver. While the brake and steering interconnected in the upper layer controller (UPC), the simulation results demonstrated that this multilayer control structure guarantees the path tracking with small error. Researchers introduced an AARB including four electronic servo-valve hydraulic actuator to actively control a heavy vehicle by solving a LQ optimization problem where the front steering considered to be an uncertain disturbance [42]. The simulation results in the frequency and time domains confirmed a remarkable improvement in terms of rollover stability. 
A novel roll stability control strategy for heavy vehicles, termed dynamic game theory-based path following active anti-roll (AAR) interactive shared control strategy was proposed in [43]. It was indeed a cooperative path followingroll stability controller that had two players, i.e. AARB and AS, determined via a closed-loop feedback Nash equilibrium theory. Simulations based on various driving scenarios were conducted to investigate the effectiveness of the method.

\section{SENSOR TECHNOLOGY FOR AUTONOMOUS AHVS}

The way by which an autonomous vehicle learns about its environment is through its sensors. Sensors should be capable of providing both perceptive and locational view of the environment so that the vehicle can make decisions in realtime. Sensors are utilized to detect roads, traffic participants including vehicles and pedestrians, obstacles through their paths and the peripheral environment. In most situations these sensors should be capable of determining the distance between the vehicle and other adjacent objects as well as their relative velocities.

Generally, there is no difference in type of sensors used for passenger vehicles and AHVs. Differences are mostly related to the arrangement of sensors and their number. For instance, one of the differences is the spots on the body of vehicles in which the sensors should be embedded because of the variety in size, application and level of autonomy of different classes of vehicles. There are many types of sensors designed to be used in autonomous driving, some of them are more common which can be classified in two main categories as demonstrated in Figure 9.

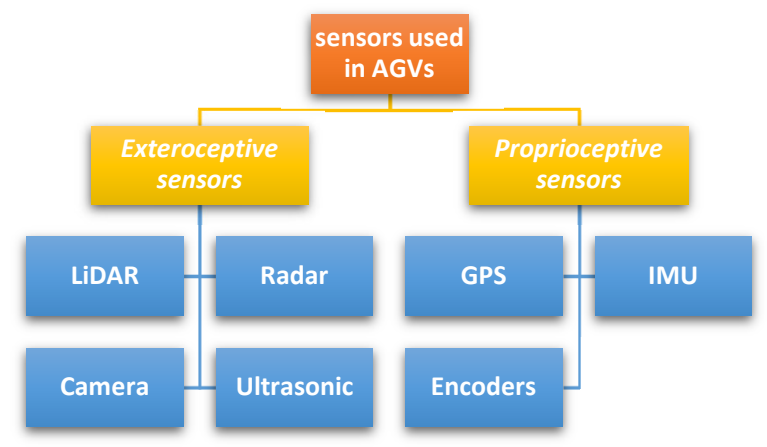

Figure 9. Main sensors employed in automated driving

LiDAR (Light Detecting \& Ranging) is a surveying method that measures distance to a target by illuminating the target with laser light and measuring the reflected light with a sensor. Differences in laser return times and wavelengths can then be used to make digital 3-D representations of the target. A significant parameter of LiDAR sensors is the distance range which they can detect to recognize the objects. Weather condition, e.g., level of humidity and the reflectivity level of object, affects the range detection considerably [44]. Today's LiDAR sensors are capable of measuring distances at rates greater than 150 kilohertz (150,000 pulses per second) and would be classified as a long-range sensor with a range of over $250 \mathrm{~m}$ or short range [45].

The full potential of these sensors has not been fully explored yet due to their high cost and low availability. They are complex mechanical mirror systems that offer full $360^{\circ}$ visibility and can cost a fortune. Today, there is a shift towards the development of lower cost LiDAR sensors that are more appropriate for extensive use. For example, solid-state LiDAR and infrared LiDAR [46]. Figure 10 illustrates sensor technologies including LiDAR, which can be used to make an AHV capable of driving itself.

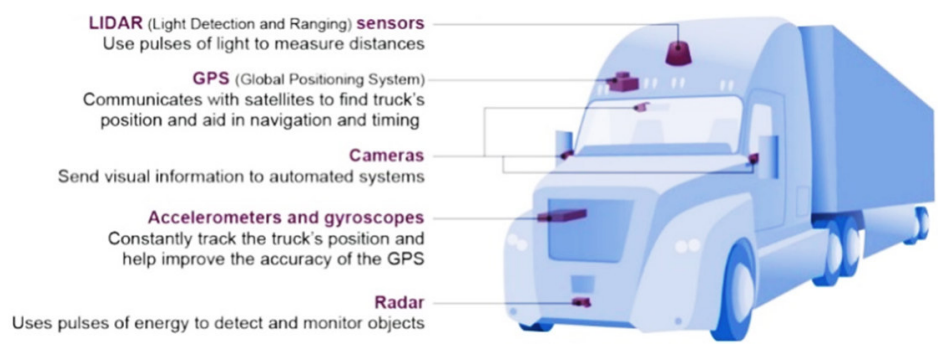

Figure 10. Sensor technologies which can be used in autonomous AHV [47]

Radar is a technology that uses electromagnetic radiation to measure the distance, angle and velocity of objects. It works on the principle of radio frequencies that can be used in several frequency bands. (e.g. $24 \mathrm{GHz}, 79 \mathrm{GHz}$ ) [46]. Higher resolution of detection is achieved by higher frequencies, and it allows the system to differentiate among many objects in the environment. Unlike LiDAR, radar has the virtue of being less affected by weather condition [44].

Radar sensors are classified in short-range to mid-range from $50 \mathrm{~m}$ to $100-150 \mathrm{~m}$ and long-range capable of detecting objects in $250 \mathrm{~m}$ distance [46]. As these kinds of sensors are much cheaper than LiDAR ones and able to detect objects in all weather conditions, radar sensors are more suitable for autonomous driving purposes. However, their small field of view (FOV), which is usually $45^{\circ}$, is deemed as a negative point compared with LiDAR. The important benefit of radars is that they are capable of determining the relative velocity of the observed objects which is advantageous for automatic braking or adaptive cruise control purposes in AGVs.

A camera works based on passive light sensors to produce a digital image of a covered region of space. Cameras can detect both moving and static objects within their surroundings. One of the major capabilities of cameras is that they can identify colors and textures. This can increase the autonomous driving perception. They are also cheap and easily accessible. The negative point of this sensor is that they are sensitive to adverse weather conditions and low intensity light. In addition, since the image is $2 \mathrm{D}$, recognizing distance to an object using the image can only be determined by employing complex processing algorithms [46]. Table 2 presents a comparison between major sensing technologies of exteroceptive environment perception.

Despite the drawbacks of cameras, in a 2019 conference, TESLA announced that they have found very successful methods (a combination of deep learning algorithms, artificial intelligence and powerful GPUs) to use just a few cameras in order to efficiently recognize the vehicle surrounding environment instead of using very expensive LiDAR or Radar sensors. They believe that the next generation of autonomous vehicles just need cameras mounted at different spots of vehicle body and use the image processing and deep learning 
to teach the central processor unit for making the best decision in every specific driving situation.

TABLE II. COMPARISON BETWEEN FUNCTIONALITIES OF MAJOR SENSOR TECHNOLOGIES [44]

\begin{tabular}{|l|c|c|c|c|}
\hline \multicolumn{1}{|c|}{ Criteria } & Radar & LiDAR & Ultrasound & Camera \\
\hline $\begin{array}{l}\text { Very short-range } \\
\text { detection (up to } 1 \mathrm{~m})\end{array}$ & ok & Poor & Very good & Ok \\
\hline $\begin{array}{l}\text { Short-range detection } \\
(1-30 \mathrm{~m})\end{array}$ & Very good & Very good & Poor & Good \\
\hline $\begin{array}{l}\text { Long-range detection } \\
(30-100 \mathrm{~m})\end{array}$ & Very good & Medium & No & Poor \\
\hline Angle $<10^{\circ}$ & Good & Very good & Poor & Good \\
\hline Angular resolution & Good & Very good & Poor & Good \\
\hline Velocity measurement & Yes & No & No & No \\
\hline Poor weather condition & Very good & Poor & Good & Poor \\
\hline Night & Very good & Very good & Very good & limited \\
\hline
\end{tabular}

GPSs (satellite-based radio-navigation systems) are common sensors for geolocation and navigation. Inertial Measurement Units (IMUs) are electronic devices that can measure a body's force, angular velocity and magnetic field. IMUs combined with encoders are electro-mechanical devices that can convert linear or angular position of a shaft to an analogue or digital signal. GPSs, IMUs and encoders are three vital sensors to help vehicles to drive autonomously.

\section{CHALlENGES AND FUTURE DIRECTIONS}

AHVs and especially multi-trailer AHVs (MTAHVs) are increasingly used around the world due to their economic and environmental benefits. Despite the benefits, MTAHVs exhibit poor maneuverability and low lateral stability compared to the heavy trucks and passenger vehicles due to their multi-unit structures, large sizes and high centers of gravity[48]. Past studies on semi-autonomous and autonomous driving used simple driver and yaw-plane vehicle models [5][9][14]. To simulate MTAHV dynamics effectively and to predict driver behaviors accurately, computationally efficient yaw-roll vehicle models and driver models considering the motion cues of trailing units need to be derived. To date, rare systematic threat assessment has been conducted for MTAHV motion planning in high-speed operations, and in the case of MTAHVs almost no research has been done. The multi-unit structures and roll dynamics of MTAHVs imposes more challenges in threat assessment and path planning compared against the cases of passenger cars [49]. Past studies on autonomous driving for articulated vehicles focused on automatic steering without automated braking [50]. To design autonomous driving for AHVs in highway operations, it is reasonable to take into account both automatic steering and braking. A study has been conducted to identify distinct AHV dynamic characteristics between manual and automated driving in lane changes, but the interactions of driver-automation-environment in automation intervention have not been examined [51].

\section{CONCLUSIONS}

Contemporary progresses in autonomous vehicles yield bright future autonomous driving. Official future prognoses about autonomous vehicles point out that a vast number of automotive companies will launch products with semi and fully autonomous features in near future. It is expected that by
2035 the majority of vehicles including heavy commercial vehicles will be fully automated. This paper reviews the historical and current developments of automated driving features and necessary technologies with a focus on AHVs. It is disclosed that little attention has been paid to exploring autonomous driving for AHVs and, in particular, MTAHVs. In the limited studies on automotive driving for AHVs, the threat assessment due to the poor maneuverability and low lateral stability of these large vehicles has not been addressed.

\section{REFERENCES}

[1] Z. Ni and Y. He, "Design and validation of a robust active trailer steering system for multi-trailer articulated heavy vehicles," Veh. Syst. Dyn., vol. 3114, 2018.

[2] "U.S. Federal Motor Carrier Safety Administration. Large Trucks and bus crash facts 2016." [Online]. Available: https://cms8.fmcsa.dot.gov/safety/data-and-statistics/large-truck-andbus-crash-facts-2016\#A8.

[3] OECD/ITF, Road Safety Annual Report 2015. 2015.

[4] P. GUTOSKIE, Road Safety in Canada, vol. 25, no. 1. 2001.

[5] Standing Senate Committee on Transport and Communications Senate, "Driving Change-Technology and Future of Autonomous Vehicles," no. January, 2018.

[6] P. Nilsson, Traffic Situation Management for Driving Automation of Articulated Heavy Road Transports From driver behaviour towards highway autopilot. 2017.

[7] C. Cheng and D. Cebon, "Improving roll stability of articulated heavy vehicles using active semi-trailer steering," Veh. Syst. Dyn., vol. 46, no. SUPPL.1, pp. 373-388, 2008.

[8] World Health Organization. GLOBAL STATUS REPORT ON ROAD SAFETY, no. 1. 2018.

[9] K. Bengler, K. Dietmayer, B. Färber, M. Maurer, C. Stiller, and H. Winner, "Three Decades of Driver Assistance Systems," IEEE Intell. Transp. Syst. Mag., vol. 6, no. 4, pp. 6-22, 2014.

[10] A. T. Van Zanten, "Bosch ESP systems: 5 years of experience," $S A E$ Tech. Pap., no. 724, 2000.

[11] S. J. Anderson, S. B. Karumanchi, K. Iagnemma, and J. M. Walker, "The Intelligent CoPilot," IEEE Intell. Transp. Syst. Mag., no. April 2013, pp. 45-54, 2013.

[12] V. A. Shia et al., "Semiautonomous vehicular control using driver modeling," IEEE Trans. Intell. Transp. Syst., vol. 15, no. 6, pp. 26962709, 2014.

[13] A. Gray, Y. Gao, T. Lin, J. K. Hedrick, and F. Borrelli, "Stochastic predictive control for semi-autonomous vehicles with an uncertain driver model," IEEE Conf. Intell. Transp. Syst. Proceedings, ITSC, no. 1239323, pp. 2329-2334, 2013.

[14] SAE International Surface Vehicle Information Report, "Taxonomy and Definitions for Terms Related to On-Road Motor Vehicle Automated Driving Systems," SAE Standard J3016, 2016.

[15] N. Christie, A review of accidents and injuries to road transport drivers. 1831.

[16] B. J. Alshaer, T. T. Darabseh, and M. A. Alhanouti, "Path planning, modeling and simulation of an autonomous articulated heavy construction machine performing a loading cycle," Appl. Math. Model., vol. 37, no. 7, pp. 5315-5325, 2013.

[17] A. Elhassan, "Autonomous driving system for reversing an articulated vehicle SCHOOL OF ELECTRICAL ENGINEERING," 2015.

[18] P. F. Lima, Optimization-Based Motion Planning and Model Predictive Control for Autonomous Driving: With Experimental Evaluation on a Heavy-Duty Construction Truck. 2018.

[19] S. Taheri, "Steering Control Characteristics of Human Driver Coupled with an Articulated Commercial Vehicle," no. January, 2014.

[20] S. Siregar, "A New Design of Human-Machine Interaction A Leap Towards Safe Driving," 2015. 
[21] N. H. Amer, H. Zamzuri, K. Hudha, and Z. A. Kadir, "Modelling and Control Strategies in Path Tracking Control for Autonomous Ground Vehicles: A Review of State of the Art and Challenges," J. Intell. Robot. Syst. Theory Appl., vol. 86, no. 2, pp. 225-254, 2017.

[22] P. Fancher and C. Winkler, "Directional performance issues in evaluation and design of articulated heavy vehicles," User Model. User-adapt. Interact., vol. 45, no. 7-8, pp. 607-647, 2007.

[23] "Difference between A B C Trains." [Online]. Available: https://www.bigtruckguide.com/get-your-a-b-cs-straight/. [Accessed: 23-Oct-2019].

[24] M. S. Kati, "Definitions of Performance Based Characteristics for Long Heavy Vehicle Combinations Prepared by: Improving landfill monitoring programs with the aid of geoelectrical - imaging techniques and geographical information systems," 2013.

[25] M. Gäfvert and O. Lindgärde, "A 9-DOF tractor-semitrailer dynamic handling model for advanced chassis control studies," Veh. Syst. Dyn., vol. 41, no. 1, pp. 51-82, 2004.

[26] M. M. Islam, X. Ding, and Y. He, “A closed-loop dynamic simulationbased design method for articulated heavy vehicles with active trailer steering systems," Veh. Syst. Dyn., vol. 50, no. 5, pp. 675-697, 2012.

[27] M. M. Islam and Y. He, "A parallel design optimisation method for articulated heavy vehicles with active safety systems," Int. J. Heavy Veh. Syst., vol. 20, no. 4, pp. 327-341, 2013.

[28] S. Cheng, L. Li, H.-Q. Guo, Z.-G. Chen, and P. Song, "Longitudinal Collision Avoidance and Lateral Stability Adaptive Control System Based on MPC of Autonomous Vehicles," IEEE Trans. Intell. Transp. Syst., vol. PP, pp. 1-10, 2019.

[29] D. J. Fagnant and K. Kockelman, "Preparing a nation for autonomous vehicles: Opportunities, barriers and policy recommendations," Transp. Res. Part A Policy Pract., vol. 77, pp. 167-181, 2015.

[30] J. Manyika, M. Chui, and J. Bughin, "Disruptive technologies: Advances that will transform life, business, and the global economy," McKinsey Glob. ..., no. May, p. 163, 2013.

[31] A. Khodayari, A. Ghaffari, S. Ameli, and J. Flahatgar, "A historical review on lateral and longitudinal control of autonomous vehicle motions," ICMET 2010 - 2010 Int. Conf. Mech. Electr. Technol. Proc., no. Icmet, pp. 421-429, 2010.

[32] H. V. Systems, "Comprehensive path and attitude control of articulated vehicles for varying vehicle conditions Hsin Guan, Kyongil Kim and Bo Wang*," vol. 24, no. 1, 2017.

[33] [33] K. il Kim, H. Guan, B. Wang, R. Guo, and F. Liang, "Active steering control strategy for articulated vehicles," Front. Inf. Technol. Electron. Eng., vol. 17, no. 6, pp. 576-586, 2016.

[34] H. Yuan and H. Zhu, "Anti-jackknife reverse tracking control of articulated vehicles in the presence of actuator saturation," Veh. Syst. Dyn., vol. 54, no. 10, pp. 1428-1447, 2016.

[35] B. A. Jujnovich and D. Cebon, "Path-following steering control for articulated vehicles," J. Dyn. Syst. Meas. Control. Trans. ASME, vol. 135 , no. 3, pp. 1-15, 2013.

[36] A. Goodarzi and M. Ghajar, "Integrating lane-keeping system with direct yaw moment control tasks in a novel driver assistance system," Proc. Inst. Mech. Eng. Part K J. Multi-body Dyn., vol. 229, no. 1, pp. 16-38, 2015.

[37] M. M. Michałek, "A highly scalable path-following controller for Ntrailers with off-axle hitching," Control Eng. Pract., vol. 29, pp. 6173, 2014.

[38] T. Nayl, G. Nikolakopoulos, T. Gustafsson, D. Kominiak, and R. Nyberg, "Design and experimental evaluation of a novel sliding mode controller for an articulated vehicle," Rob. Auton. Syst., vol. 103, pp. 213-221, 2018

[39] P. Gaspar, I. Szaszi, and J. Bokor, "The design of a combined control structure to prevent the rollover of heavy vehicles," Eur. J. Control, vol. 10, no. 2, pp. 148-162, 2004.

[40] S. Yim, K. Jeon, and K. Yi, "An investigation into vehicle rollover prevention by coordinated control of active anti-roll bar and electronic stability program," Int. J. Control. Autom. Syst., vol. 10, no. 2, pp. 275 $287,2012$.
[41] M. Ghazali, M. Durali, and H. Salarieh, "Path-following in model predictive rollover prevention using front steering and braking," Veh. Syst. Dyn., vol. 55, no. 1, pp. 121-148, 2017.

[42] V. T. Vu, O. Sename, L. Dugard, and P. Gaspar, "Active anti-roll bar control using electronic servo valve hydraulic damper on single unit heavy vehicle," IFAC-PapersOnLine, vol. 49, no. 11, pp. 418-425, 2016.

[43] Y. Liu, K. Yang, X. He, and X. Ji, "Active steering and anti-roll shared control for enhancing roll stability in path following of autonomous heavy vehicle," SAE Tech. Pap., vol. 2019-April, no. April, pp. 1-10, 2019.

[44] C. Ilas, "Electronic sensing technologies for autonomous ground vehicles: A review," 2013 - 8th Int. Symp. Adv. Top. Electr. Eng. ATEE 2013, pp. 0-5, 2013

[45] C. Ilas, "Perception in autonomous ground vehicles: A review," 2013 Int. Conf. Electron. Comput. Artif. Intell. ECAI 2013, no. June 2013, 2013.

[46] S. Campbell et al., "Sensor Technology in Autonomous Vehicles: A review," 29th Irish Signals Syst. Conf. ISSC 2018, pp. 1-4, 2018.

[47] R. Agencies and U. S. Senate, "AUTOMATED Federal Agencies Should Take Additional Steps to Prepare for Potential Workforce Effects," 2019.

[48] Y. He, M. M. Islam, S. Zhu, and T. Hu, “A design synthesis framework for directional performance optimization of multi-trailer articulated heavy vehicles with trailer lateral dynamic control systems," Proc. Inst. Mech. Eng. Part D J. Automob. Eng., vol. 231, no. 8, pp. 1096-1125, 2017.

[49] T. Nayl, DOC TOR A L T H E S I S On Autonomous Articulated Vehicles Thaker Nayl, no. June 2015. 2017.

[50] N. And En, A. G. Martín, K. Hoogendijk, L. Niklasson, F. Sandblom, and F. S. Seholm, "Predictive Control for Autonomous Articulated Vehicles," 2017.

[51] P. Nilsson, L. Laine, and B. Jacobson, "A Simulator Study Comparing Characteristics of Manual and Automated Driving during Lane Changes of Long Combination Vehicles," IEEE Trans. Intell. Transp. Syst., vol. 18, no. 9, pp. 2514-2524, 2017. 